\title{
Wave-based control of planar motion of beam-like mass-spring arrays
}

\author{
Hossein Habibi ${ }^{a *}$, William O'Connor ${ }^{\text {b }}$ \\ ${ }^{a}$ Department of Mechanical Engineeing, School of Engineering, Persian Gulf University, \\ Bushehr75168, Iran. \\ ${ }^{\mathrm{b}}$ UCD School of Mechanical and Materials Engineering, Belfield, Dublin4, Ireland.
}

\begin{abstract}
Wave-based control (WBC) is a simple and relatively new technique for motion control of under-actuated flexible systems. To date it has been mainly applied to rectilinear lumped flexible systems. The current work focuses on a development of WBC to control two-dimensional beamlike structures in which an actuator, attached to one end, acts to translate and rotate the structure through an arbitrary path in the plane. In this work, first a lumped model of a beam is developed using mass-spring arrays. The lumped beam model is of interest here as a benchmark control challenge. It can also be considered as a model of various lumped or distributed mass structures. To check the latter, the mode shapes and frequencies are first compared with those of classical beam theory. This involved a new technique to find mode shapes and frequencies for arrays. The control strategy is then presented and tested for a range of manoeuvres. As a system to be controlled, the mass-spring array presents many challenges. It has many degrees of freedom, many undamped vibration modes, is highly under-actuated, and sensing of system states is difficult. Despite these challenges, WBC performs well, combining a fairly rapid response with active vibration damping and zero steady-state error. The controller is simple to implement and of low order. It does not need or use any system model and is very robust to system changes.
\end{abstract}

\section{Keywords:}

Wave-based control, active vibration damping, control of flexible systems, beam modelling.

\section{Introduction}

To date wave-based control (WBC) has been applied mainly to controlling simultaneously the motion and vibration of chain-like dynamical systems, with inertial and elastic elements in series, to represent e.g. robot arms, antenna systems, solar panel arrays, gear trains or flexural beams, whether these are translating, rotating and slewing [1-5]. Among the many methods that have been applied to single-actuator, position control of lumped or distributed flexible mechanisms (see [6-11], for example), $W B C$ has shown itself to be a very effective, robust, generic and low-cost control strategy.

This paper investigates the extension of the technique to control more complex flexible systems. The focus is on beam-like structures in two dimensions, modelled by arrays of lumped, point masses and interconnecting springs, as shown in Fig.1a. The diagonal springs within the rectangular array are needed to add some shear stiffness: without them the system would tend to collapse. Although such arrays could be considered simply for the interesting control challenge they present, they can also model various predominantly lumped structures of engineering interest, ranging from small micro-electromechanical devices, micro-surgical tools, through

\footnotetext{
* Corresponding author: e-mail: habibi@pgu.ac.ir
} 
manipulators and robot arms, up to large space structures. In addition, one may consider them as discretised models of distributed systems, such as beams. Then, by choosing suitable mass and spring values, they can model distributed systems of given mass densities and elastic constants, uniform or not.

An obvious challenge is that such systems have many degrees of freedom, with many undamped vibration modes and natural frequencies. Furthermore they are clearly highly underactuated. There is a single actuator at one end (AB in Fig. 1a), directly connected to only a few masses (at least two), which is attempting to translate and rotate the entire system to a new position and orientation in the plane. In other words, there is a target angular displacement, $\theta$, in addition to target translational $x$ and $y$ displacements, all to be achieved by the controlled motion of a single actuator.

Wave-based control sees the actuator motion as launching mechanical waves into the flexible system, while absorbing waves coming back from the system. In the 1-D rectilinear case, with a simple string of masses and springs, the displacement waves have a single, two-way path, from actuator to system tip and back again. This single wave path also helps limit the wave dispersion (although some dispersion is always present) [12, 13]. In the 2-D system, by contrast, there are multiple, complex wave paths from the actuator to the various system boundaries and back again, so the dispersion and wave mixing are even more noticeable. A further complication is coupling between transverse (shearing / rotational) motion and longitudinal motions in different directions throughout the grid. It was not clear beforehand, therefore, whether the $W B C$ ideas for controlling such a system would work, and, if so, how well.

\section{The flexible system model}

The dynamic system of masses and springs in the 2-D array was simulated by a straightforward computation scheme, involving a direct application of Newton's second law of motion and Hooke's law. The magnitudes and directions of all the forces on each mass were determined from its own position, the positions of its neighbouring masses (or of the actuator itself for some of the masses), combined with the known spring stiffness values. A typical internal mass experienced forces from a maximum of five neighbouring masses. The resulting accelerations were then integrated to get the new velocities and again to get the new positions, from which forces could be determined for the next integration step. In this work all the numerical modeling was implemented using the commercial software Matlab and its associated control toolbox, Simulink.

In the models both external damping (viscous damping to ground) and internal damping (viscous damping proportional to relative velocities between masses) were included. For the results presented below, however, all damping was set to zero, so that the only source of vibration damping was the active damping due to the control system in the actuator. A real system typically has internal damping. However, in order to face the most challenging case and the worst possible scenario to control, no damping is considered here. As it was observed, but not shown here, for adding trivial internal damping, the system settled at target and residual vibrations died out even more rapidly than shown in the figures in this work. Also gravity was not considered in the work presented here, so that the 2D system can be considered to be moving in a horizontal plane, or in a micro-gravity environment.

All motion was initiated by moving the actuator. Initially the actuator was assumed to be ideal, so that the actual actuator motion was made equal to the reference actuator motion. Later realistic actuator dynamics were added, which included the effects of dynamic loads due to the attached flexible system. In the second case the actuator was assumed to have its own sub- 
controller, responsible only for ensuring that the actuator's actual motion followed the reference actuator motion as closely as possible. Again in the case of $2 \mathrm{D}$ systems, the $W B C$ was at a higher level, at which the details of this sub-controller were of no concern.

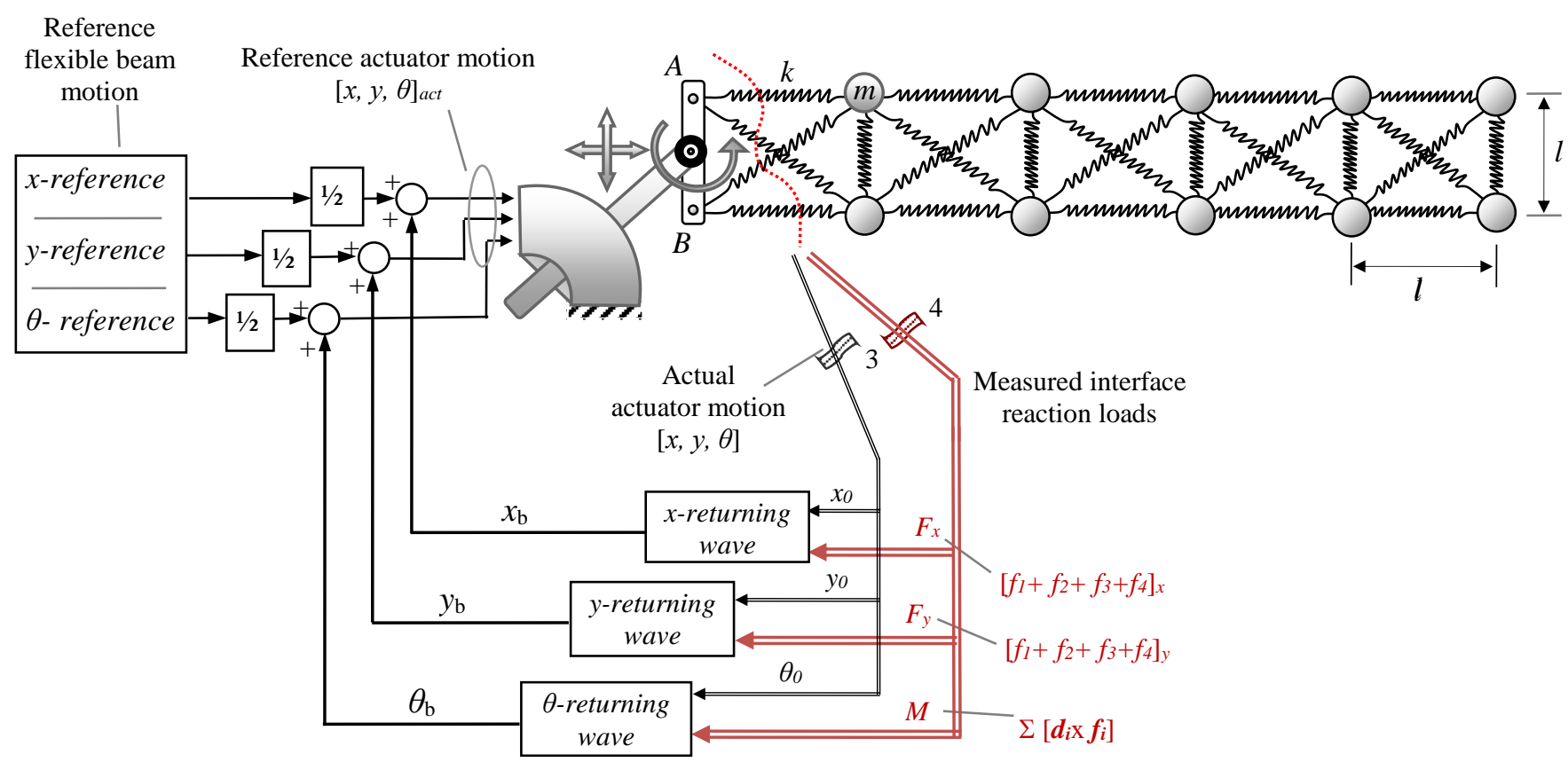

(a)

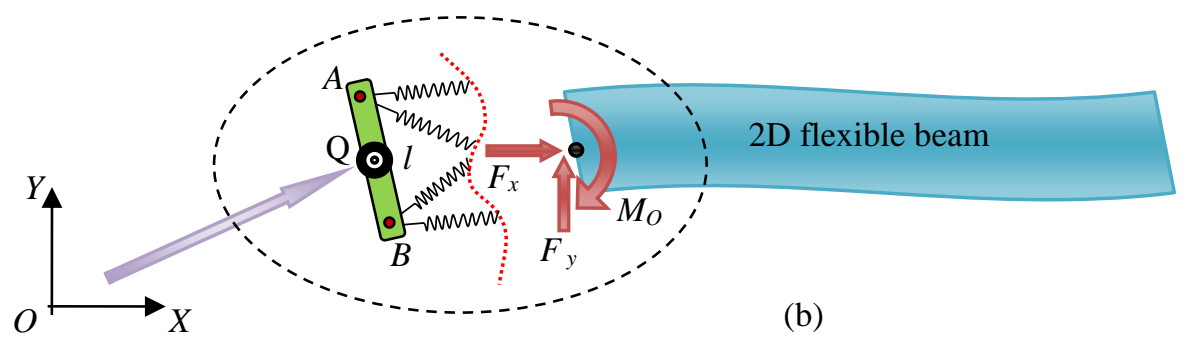

Fig. 1: A representation of the beam model; a) WBC of a flexible beam modelled as a mass-spring array; b) interface details shown as the equivalent loads applied on the beam from the actuator through a typical maneuver

The array can be given arbitrary shapes and sizes, with arbitrary values of masses and springs. In the present work the focus was on a beam model with a bar-like actuator (Fig. 1a) representing, for example, a beam-like flexible robot arm or manipulator.

There is considerable work in the literature on controlling flexible beams. In much of this work only vibration control has been considered (e.g. [14-18]), without considering the question of re-positioning (gross motion, whether translation or rotation). Even with the vibration control problem, some people such as Mace \& Jones [14] assumed only steady state vibrations/waves and at only one frequency, so the control scheme cannot deal with transients. Papers which do consider gross beam motion, as well as vibration, focus almost inclusively on rotation around a fixed hub. Invariably, the gross motion is considered as separable from the vibration motion, effectively ignoring the dynamic coupling between them and the fact that the reference frame for the vibration analysis is accelerating and therefore not inertial. No literature was found which studied the combined translation, rotation and active vibration damping of flexible structures, 
whether by a single actuator or even using multiple actuators, or which properly treated the errors involved in notionally separating rigid and vibrational motion.

Before considering the control, the question of how closely the lumped mass-spring array can model a distributed beam will now be assessed. In this work, a beam of height $l$ and length $5 l$ was considered to act as a test bed for the application of WBC adapted for a beam-like, 2D structure. Obviously choice of an arbitrary rectangular geometry and any other set of properties in the model such as the number of masses and springs' stiffness represent a physical distributed structure anyway. But there might be this question that, for example, how many masses determining modes of vibration can constitute a good approximation for a finite distributed structure. Although this could be studied under a different topic on its own, but one may refer to the literature discussing the proximity of a lumped model to a structure through determining the number of vibration modes (see [19], for example).

\section{Two-dimensional array models: parameterisation, development and assessment}

Although the main emphasis of this paper is on the performance of WBC applied to 2D flexible beam-like structures, one might query the extent to which the arrays of lumped masses and springs could model real structures, especially those with distributed material properties. The lumped model was chosen for its adaptability, ease of implementation, its avoidance of treating rigid and flexible motion in different ways, and its claim to represent in a generic way a range of systems of importance in engineering. Some systems and structures have obviously lumped characteristics, so the appropriateness of a lumped model is then fairly clear. Examples include solar panel arrays on satellites and robots with rigid arms and flexible joints. But other systems are clearly better considered as having distributed properties. In particular, beams are widely used to model flexible robot arms, for example, but also as basic elements within more complex structures. So while it has been shown in some detail how WBC can control beam-like arrays, there is the remaining doubt whether this is a valid test for real beams, or for beams represented by more conventional modeling assumptions, such as Euler-Bernoulli beam theory or Timoshenko beam theory.

Euler-Bernoulli beam theory makes certain simplifying assumptions within the theory of linear elasticity to obtain a differential equation for the beam deflection. It covers the case of small deflections of thin beams subjected to lateral loads only, in which shear deformation can be ignored [20]. It is thus a special case of the more developed Timoshenko beam theory, which accounts for shear deformation and rotational inertia effects, making it suitable for describing the behavior of short and thick beams, sandwich composite beams or beams subjected to highfrequency excitation when the wavelength approaches the thickness of the beam. If such beams can be modelled by the lumped array, the question remains regarding how to choose the mass and spring values to model a given beam of specified dimensions and material properties, including, its values for Young's modulus $(E)$, shear modulus $(G)$, Poisson's ratio $(v)$ and density $(\rho)$.

In this section, the flexible, beam-like array shown in the upper right of Fig. 1a is characterized using classical mechanics of materials static relationships, such as Hooke's law. Then its dynamic, elastic behavior will be assessed by comparing its natural frequencies and mode shapes with those of a distributed cantilever beam, for both longitudinal and lateral vibrations. 


\subsection{Analytical approach}

Numerical models of distributed mechanical systems involve discretising the system in one way or another, so that they can always be considered to be "lumped" in some sense. It could also be argued that, at a sufficiently fine scale (down to molecular levels), distributed systems are also lumped. But at larger scales of general engineering interest, this lumped nature is not relevant.

In engineering generally, the Finite Element Method (FEM) is now a standard technique for modelling and discretising distributed systems. There is considerable literature on the topic, such as by Vasques \& Rodrigues [15], Gams et al [21], and Cohodar et al [22]. Finite difference approaches have also been widely used.

Regarding the 2D mass-spring arrays used in this work, there is only a small amount of published work. For example, a two-dimensional lumped model for deformable continuums has been developed by Williams [23]. His model consists of particles connected to each other via extensional and rotational springs. He presented the spring constants of the model in terms of the material properties (Lame constants) of the body being modelled using the equations of motions of the elastic continuum from continuum mechanics. Here we use a classical mechanical approach to establish a relationship between the grid masses and spring constants in different directions within the grid and the corresponding material properties used in continuum mechanics. It is assumed that grid deformations remain small.

Figure 2a depicts a basic cell of the mass-spring array making up the beam model. It is stretched longitudinally by applying an axial force $(F)$, as shown in the free-body diagrams in Figs.2b, c. The sprung unit is assumed to be stretched by $\Delta l_{x}$ horizontally and compressed by $\Delta l_{y}$ vertically.

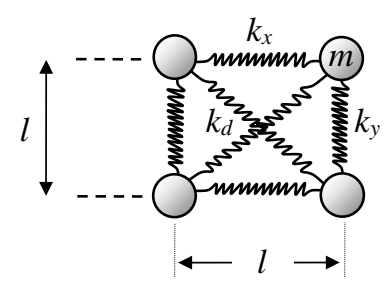

(a)

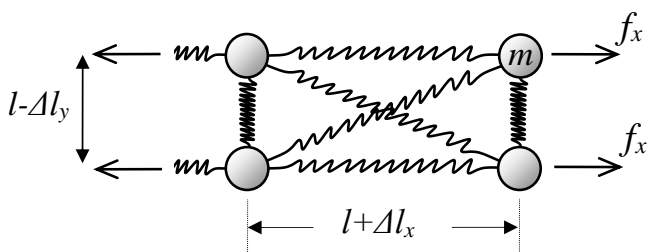

(b)

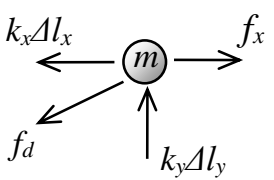

(c)

Fig. 2: The far end unit of the lumped beam-like structure shown in Fig.1a; a) unloaded state; b) freebody diagram of the stretched unit; c) free-body diagram of the top, right mass of the stretched model.

In the figure, $k_{x}, k_{y}$ and $k_{d}$ are spring stiffness in the $x, y$ and diagonal directions respectively. Assuming small deflections and so small angle changes in the diagonal direction, the correspondent force $\left(f_{d}\right)$ in the diagonal spring is approximately

$$
f_{d}=k_{d} \sqrt{\Delta l_{x}^{2}+\Delta l_{y}^{2}}
$$

Also, the static equilibrium of the mass shown in Fig. $2 \mathrm{c}$ in the $x$ - $y$ directions yields

$$
\begin{gathered}
f_{x}=k_{x} \Delta l_{x}+\frac{1}{\sqrt{2}} k_{d} \sqrt{\Delta l_{x}{ }^{2}+\Delta l_{y}{ }^{2}} \\
k_{y} \Delta l_{y}=\frac{1}{\sqrt{2}} k_{d} \sqrt{\Delta l_{x}{ }^{2}+\Delta l_{y}{ }^{2}}
\end{gathered}
$$

Among the material mechanical properties, Young's modulus, $E$, is perhaps the most important. It can be calculated by dividing the tensile stress by the tensile strain in the elastic (initial, linear) portion of the stress-strain curve: 


$$
E=\frac{\sigma}{\varepsilon}=\frac{F l}{A \Delta l_{x}}
$$

where $F$ is the force per unit length exerted on the sample unit under tension i.e. $F=2 f_{x}$. Also assuming a cross section area per unit width as $A=l \times 1$, Eq. (4) is simplified to

$$
E=\frac{F}{\Delta l_{x}}
$$

Likewise Poisson's ratio, $v$, which is the ratio of lateral to longitudinal strain, can be calculated as

$$
v=-\frac{\Delta l_{y}}{\Delta l_{x}}=-\frac{k_{d}}{\sqrt{2} k_{y}} \sqrt{1+\left(\frac{\Delta l_{y}}{\Delta l_{x}}\right)^{2}}
$$

Ignoring the second order term it can be approximated as

$$
v=-\frac{k_{d}}{\sqrt{2} k_{y}}
$$

Now, substituting Eq.(2) into Eq.(5) and considering Eq.(6), the Young's modulus, E, is given by

$$
E=2 k_{x}+\frac{k_{d}}{k_{y}} \sqrt{2 k_{y}^{2}+k_{d}^{2}}
$$

For Euler-Bernoulli beams only $E$ is required, but for Timoshenko beams the shear modulus, $G$, is also needed. These two moduli $(E$ and $G$ ) are related through Poisson's ratio as follows:

$$
G=\frac{E}{2(1+v)}
$$

Therefore replacing Eqs. (7) and (8) into Eq. (9) after simplification yields

$$
G=\frac{\sqrt{2} k_{x} k_{y}}{\left(\sqrt{2} k_{y}-k_{d}\right)}+\frac{\sqrt{2} k_{d}}{2\left(\sqrt{2} k_{y}-k_{d}\right)} \sqrt{2 k_{y}{ }^{2}+k_{d}{ }^{2}}
$$

Now for a given beam with given material elastic properties $E, v$ and $G$, appropriate model stiffness values, $k_{x}, k_{y}$ and $k_{d}$, can be determined using Eqs. (7), (8) and (10). Alternatively, going the other way, given array stiffness values can be converted to approximate distributed elastic parameters. Finally, the lumped mass values can be related to the given material density, the given beam dimensions, and the number of masses in the lumped model.

These relationships were based on a static analysis. The next section considers the dynamic behavior of the lumped array and how well it corresponds to that of distributed beam theory.

\subsection{Modal analysis using WBC}

In attempting to compare the dynamic behaviour of the beam-like array with that of, say, a cantilever beam, for which there are precise, analytical solutions for natural frequencies and mode shapes, a problem arises. How can the mass-spring array be made to vibrate at a single resonant frequency and mode? At resonance, the whole system should be moving synchronously (all masses moving at one frequency) with a definite mode shape (the ratios of amplitudes of 
oscillations of masses constant over time). But it is a challenge to achieve this, whether by a theoretical-analytic approach or using numerical simulation. If the mode shape is known exactly, then the system could be initialised with that shape, released from rest, and it should then vibrate synchronously. But the mode shape is not known beforehand. It would consist of specific $x$ and $y$ initial displacements of each on $n$ masses, all by different, unknown amounts. Alternatively, if the resonance frequency is known, or could be searched for, the system could be driven at this frequency. But it is not known, and even if it could be calculated, the system must be started with some initial condition, usually at rest, and then driven from there. There will then be a transient from these initial conditions, which in the absence of damping, will not die out, but will mix with the desired resonant frequency response.

Clearly then, with $2 n$ degrees of freedom and $4 n$ initial conditions, all unknown, getting a lumped, $n$-mass, 2-D, array to vibrate at resonance is not a trivial matter. A solution was developed based on driving the modelled array in such a way that it found the resonant conditions itself. Wave-based control was used to achieve this. For the fixed-free, cantilever case, instead of holding the intended "fixed" end of the lumped array motionless, it was initially driven harmonically, but also with a wave-based controller. In other words, the launch wave was a sinusoid of variable frequency, and the return wave was absorbed using WBC. The frequency was then slowly adjusted until frequencies were found at which the actuator became stationary. At that stage it was deduced (see explanation below) that the system was vibrating at a fixedfree resonant frequency. In the absence of damping, it continued to vibrate synchronously for an indefinite time (with no further input from the stationary actuator).

In practice, the array shown in Fig.1a was excited in either the lateral or longitudinal direction with a sinusoidal input of arbitrary amplitude and variable frequency. Obviously if no control is applied the response is erratic. If the excitation frequency $(\omega)$ of the sinusoidal input, $r=$ $a_{0} \sin (\omega t)$, exactly matches a natural frequency, $\omega_{n}$, then the actuator vibrations quickly decay to zero. In other words, the actuator becomes fixed, while the rest of the system keeps oscillating for an unlimited time, synchronously, with no further input, as a free vibration.

The situation is shown in Fig.3 for a 10-mass system. It depicts finding a resonance at the first natural frequency of the lumped beam model for an arbitrarily chosen set of spring constants $\left(k_{x}\right.$, $k_{y}, k_{d}$ ) and mass values $(m)$ due to a sinusoidal input in the lateral or $y$-direction. In this example, the oscillating curve (dotted line) shows the motion of the last mass in the 10-mass beam-like model while the solid (green) curve represents the actuator response. Clearly, the actuator comes to rest quite quickly, while the system then continues to oscillate at a constant amplitude and at a single frequency. Resonance conditions have been found.

A more exhaustive explanation of this phenomenon requires establishing a formal connection between modal analysis and wave analysis as described by O'Connor \& McKeown [24]. However a brief explanation is as follows. Under WBC, with a sinusoidal reference input, the fixed frequency launch wave enters the system. The WBC system then measures and absorbs the returning wave. If this returning wave happens to be an exact inverse of the launch wave, with the same amplitude and frequency, but inverted (or with a phase shift of $180^{\circ}$ ) then the sum of the launch wave and return wave will be exactly zero, and will stay at zero, and the actuator will stop moving (despite the continuing launch wave). This situation is often referred to as "phase closure". This will happen only at very special frequencies. The returning wave must be a pure sinusoid of the same frequency as the launch wave. In other words the system must be moving synchronously, at the launch frequency. Furthermore the system is now the equivalent of a fixedfree system, so it must be vibrating at one of the resonant frequencies. 


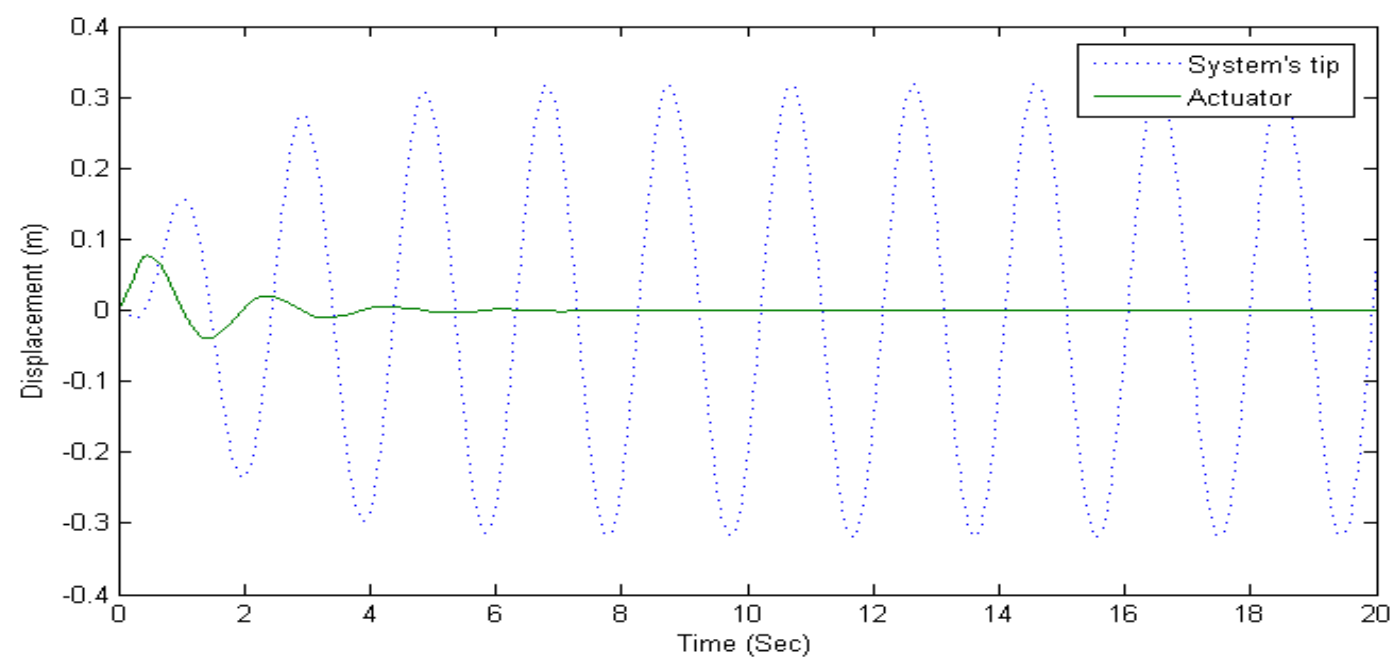

Fig. 3: Lateral oscillation of the lumped beam model under WBC when the input signal frequency is at the resonance of the first mode

During the transient stage, for example from start-up, the absorbing action of WBC serves to dampen out the vibrations happening at other frequencies, while the launch wave continues to insert the fixed frequency. If this frequency is not at a resonance, when the transient has died out there will be more or less actuator motion, as WBC moves to absorb the returning wave. It is only when the amplitude and phase are just right will the actuator stop moving, and this will happen only at one of the fixed-free resonance frequencies.

Using this method, the natural frequencies (and mode shapes) of the lumped beam model were found for lateral and longitudinal vibrations. Longitudinal vibrations, that are vibrations in the $x$-direction only, with no lateral or slewing motion, are easier than the more general case, and will be considered first. Analytical solutions are then available for the lumped case.

\subsection{Longitudinal vibration}

For longitudinal vibration of (distributed) beams or rods, the differential equation of motion is the classical wave equation,

$$
\frac{\partial^{2} u}{\partial x^{2}}=\frac{1}{c^{2}} \frac{\partial^{2} u}{\partial t^{2}}
$$

where $u$ is the $x$-displacement from the undeformed position, $c=\sqrt{\frac{E}{\rho}}$, and $\rho$ is the linear density. For a fixed-free beam, with boundary conditions $u(0, t)=0$ and $\frac{\partial u}{\partial x}(L, t)=0$, the natural frequencies of the beam can be shown to be

$$
\omega_{n}=(2 n-1) \frac{\pi c}{2 L}
$$

where $L$ is the length of the beam. For the case corresponding to the model shown in Fig.1a, $L=$ $5 l)$ and $n(=1,2, \ldots)$ is the number of the mode shape, going to infinity for the distributed case.

For the lumped case, finding the modal frequencies and shapes can be expressed as an eigenvalue-eigenvector problem. Because only $x$-motion is being considered, instead of the 10 mass, or 20-DOF system in 2-D, one may produce an equivalent, uniform 1D system with 5DOF by simply summing each pair of vertically-adjacent masses (shown in Fig. 1a) i.e. $m_{i}=$ $2 m$. Likewise the equivalent spring constant $\left(k_{i}\right)$ between every two combined masses in line, considering Fig. 2, can be calculated as 


$$
k_{i}=2 k_{x}+2 k_{d}\left(\cos \frac{\pi}{4}\right)^{2}
$$

Then to determine the 5 natural frequencies of the 5-DOF equivalent rectilinear system, the eigenvalue problem becomes

$$
M^{-1} K X=\omega_{n}^{2} X
$$

where $M$ is the $(n \times n)$ mass matrix, $K$ is the $(n \times n)$ stiffness matrix and $X$ is the eigen-vector consisting of the displacement variables. In this case, assuming the motion equations of a uniform system of 5 identical masses $\left(m_{i}\right)$ and spring constants $\left(k_{i}\right)$, these matrices are

$$
M=m_{i}\left[\begin{array}{ccccc}
1 & 0 & 0 & 0 & 0 \\
0 & 1 & 0 & 0 & 0 \\
0 & 0 & 1 & 0 & 0 \\
0 & 0 & 0 & 1 & 0 \\
0 & 0 & 0 & 0 & 1
\end{array}\right], K=k_{i}\left[\begin{array}{ccccc}
2 & -1 & 0 & 0 & 0 \\
-1 & 2 & -1 & 0 & 0 \\
0 & -1 & 2 & -1 & 0 \\
0 & 0 & -1 & 2 & -1 \\
0 & 0 & 0 & -1 & 1
\end{array}\right], X=\left[\begin{array}{l}
X_{1} \\
X_{2} \\
X_{3} \\
X_{4} \\
X_{5}
\end{array}\right]
$$

Then the correspondent natural frequencies $\left(\omega_{n}\right)$ can be obtained by solving for the eigenvalues $\left(r=\omega_{n}^{2}\right)$ to yield the following set

$$
\omega_{n}=\left\{\begin{array}{l}
0.2846 \\
0.8308 \\
1.3097 \\
1.6825 \\
1.9190
\end{array} \sqrt{\frac{k_{i}}{m_{i}}}\right.
$$

There are now three approaches to characterising longitudinal vibrations: analytical analysis for a distributed beam; modal analysis using eigenvalue-eigenvector techniques; experimentally determined values using WBC on the lumped model. The calculated natural frequencies for the three approaches are compared for three, arbitrarily-chosen, sets of 2D beam model parameters as shown in Table 1.

Table 1: Three property sets of the 2D, lumped, flexible, beam model

\begin{tabular}{|c|c|c|c|}
\hline & Set 1 & Set 2 & Set 3 \\
\hline$k_{x}(\mathrm{~N} / \mathrm{m})$ & 200 & 600 & 1000 \\
\hline$k_{\mathrm{y}}(\mathrm{N} / \mathrm{m})$ & 400 & 450 & 800 \\
\hline$k_{\mathrm{d}}(\mathrm{N} / \mathrm{m})$ & 200 & 500 & 1200 \\
\hline$l(\mathrm{~m})$ & 0.2 & 0.25 & 0.1 \\
\hline$m(\mathrm{~kg})$ & 0.3 & 0.35 & 0.4 \\
\hline
\end{tabular}

The first two modes of the system are now considered. Table 2 compares natural frequencies estimated by the three methods: $\omega_{n}$, from eigenvalues, Eq. (16); $\omega_{b}$, from the wave equation Eq. (12); and $\omega_{\mathrm{w}}$, using WBC on the array model. Young's modulus $(E)$ in $c=\sqrt{\frac{E}{\rho}}$ of Eq.(12) is determined from Eq. (8). The results suggest that even the relatively low order, 10-mass lumped model can be taken as a reasonably good approximation to a continuous beam undergoing longitudinal vibrations, at least for the important lowest vibration modes. The consistency is even better with the stiffer systems of Set 2 . The consistency would further improve with higher order lumped models, that is, by using more masses and springs for a given beam. 
Table 2: Comparing the natural frequencies of the 2D flexible beam model for its first two longitudinal modes using three methods for two different, arbitrarily-chosen, sets of properties specified in Table1 $\left(\omega_{b}\right.$; natural frequency obtained using wave equation: $\omega_{\mathrm{w}}$; natural frequency obtained using WBC experimental modal analysis: $\omega_{n}$; natural frequency obtained using eigenvalues).

\begin{tabular}{|c|c|c|c|}
\hline \multicolumn{2}{|c|}{} & Set 1 & Set 2 \\
\hline \multirow{2}{*}{$\begin{array}{c}\text { First } \\
\text { mode } \\
\text { shape }\end{array}$} & $\omega_{b}$ & 7.61 & 13.27 \\
\cline { 2 - 4 } & $\omega_{\mathrm{w}}$ & 8.31 & 13.95 \\
\cline { 2 - 4 } & $\omega_{n}$ & 8.99 & 15.14 \\
\hline \multirow{2}{*}{$\begin{array}{c}\text { Second } \\
\text { mode } \\
\text { shape }\end{array}$} & $\omega_{b}$ & 22.72 & 39.81 \\
\cline { 2 - 4 } & $\omega_{\mathrm{w}}$ & 23.96 & 39.90 \\
\cline { 2 - 4 } & $\omega_{n}$ & 25.27 & 40.22 \\
\hline
\end{tabular}

\subsection{Lateral vibration}

The Euler-Bernoulli equation for lateral beam vibrations is

$$
E I \frac{\partial^{4} y}{\partial x^{4}}+\rho A \frac{\partial^{2} y}{\partial t^{2}}=0
$$

where $y$ is the local deflection (assumed small) from the undeformed shape, $I$ is the second moment of the cross sectional area about the neutral axis, and $A$ is the cross section area of the beam. Using separation of variables, the solution is assumed to take the form of the product of a time function and a space function, or

$$
y(x, t)=T(t) . Y(x)
$$

The space part can then be shown to have the form

$$
Y(x)=A_{1} \sinh \beta_{n} x+A_{2} \cosh \beta_{n} x+A_{3} \sin \beta_{n} x+A_{4} \cos \beta_{n} x
$$

where $A_{i}(i=1,2,3,4)$ are four constants determined by boundary conditions. For the 'fixed-free' beam, the fixed end is characterized by the boundaries as $y(0, t)=0 \& \frac{\partial y}{\partial x}(0, t)=0$. The free end is specified by $\frac{\partial^{2} y}{\partial x^{2}}(L, t)=0 \& \frac{\partial^{3} y}{\partial x^{3}}(L, t)=0$ which together provide four equations through Eq. (19) for the four constants. Then, solving the frequency equation, Eq. (18), gives the natural frequencies of the beam in lateral vibrations as

$$
\omega_{n}=\left(\beta_{n} L\right)^{2} \sqrt{\frac{E I}{\rho A L^{4}}}
$$

where $\left(\beta_{n} L\right)^{2}$ is evaluated based on the boundary conditions. For the fixed-free case, $\left(\beta_{n} L\right)^{2}$ has values $\{3.52,22.03,61.70, \ldots\}$. The values of the first two natural frequencies, calculated using Eq. (20), are shown as $\omega_{b}$ in Table 3. These are compared with values, $\omega_{\mathrm{w}}$, obtained using the experimental WBC modal analysis, as described in Section 3.2. The first two modes are again evaluated. Young's modulus is determined as before.

Table 3 shows that, based on comparing resonance frequencies, there is reasonable agreement between even a fairly low-order lumped model of the beam and the analytical result for the distributed case. An exact correspondence would have been surprising. The correspondence however is sufficiently close to give confidence that the lumped model does capture the main beam dynamics as represented by Euler-Bernoulli beam theory. 
Table 3: Comparing the natural frequencies of the 2D flexible beam model for its first two lateral mode shapes using two methods for two different, arbitrarily-chosen, sets of properties specified in Table1

$\left(\omega_{b}\right.$; natural frequency from Euler-Bernoulli beam theory, $\omega_{\mathrm{w}}$; natural frequency from WBC experimental modal analysis).

\begin{tabular}{|c|c|c|c|}
\hline \multicolumn{2}{|c|}{} & Set 2 & Set 3 \\
\hline \multirow{2}{*}{$\begin{array}{c}\text { First } \\
\text { mode shape }\end{array}$} & $\omega_{b}$ & 1.91 & 2.15 \\
\cline { 2 - 4 } & $\omega_{\mathrm{w}}$ & 2.07 & 2.40 \\
\hline \multirow{2}{*}{$\begin{array}{c}\text { Second } \\
\text { mode shape }\end{array}$} & $\omega_{b}$ & 10.75 & 13.84 \\
\cline { 2 - 4 } & $\omega_{\mathrm{w}}$ & 10.94 & 14.03 \\
\hline
\end{tabular}

Note that, even as an approximation to a distributed system, it is possible that the lumped model is better than the Euler-Bernoulli model in a few respects. The lumped model allows the beam to undergo shear deformations, the effects of which are excluded from Euler-Bernoulli theory. Also, when a beam is slewing, centrifugal forces will cause stiffening of the system to lateral deformation, increasing the effective flexure stiffness. Again the lumped model will show this effect, whereas the Euler-Bernoulli beam model will not.

In any case, as already explained, the most important purpose of this work is to investigate the control aspects, independently of whether or not the model of the flexible system behaves exactly like a physical system. To this extent, it is sufficient to know that the model captures the most important flexible effects reasonably well.

\section{Control implementation}

The basic ideas about WBC, especially for 1-D rectilinear systems, have already appeared in the literature $([1,3,12])$ and will not be reproduced in detail here. In WBC in 2-D, as in 1-D, the entire control strategy focuses on the interface between the moving actuator and the flexible system. This interface is seen as a multi-directional gateway for energy and momentum transfer between the flexible system and the directly controlled actuator. See Fig. 1b. The form of control considered below corresponds to a particular representation of WBC, in which the measured variables (or sets of variables) are i) the actuator motion itself and ii) the forces (and torques) between the actuator and the flexible system. To this extent the 2-D case is similar to the 1-D case in the literature. The actuator is assumed to have its own motion (sub) control system.

The first set of measurements are the translational and rotational motions of the actuator itself, say $[x, y, \theta]$, which will probably be available from the actuator sub-control system. The second are the interface forces which correspond to the forces in four springs displayed in Fig.1a, whose $x$ and $y$ components are $F_{x}=\Sigma\left[f_{1}, f_{2}, f_{3}, f_{4}\right]_{x}, F_{y}=\Sigma\left[f_{1}, f_{2}, f_{3}, f_{4}\right]_{y}$. For rotation control, the moment of these forces is also required. This moment can be taken about a fixed point or about the mass centre of the system, with $M=\Sigma\left(d_{i} \mathrm{x} f_{i}\right)$, or the sum of the cross product of the position vectors $d_{i}$ and the force vectors $f_{i}$, with $i=1,2,3,4$. These interface forces and moments constitute actionreaction pairs for the actuator and flexible system. The forces are taken as positive when acting positively on the flexible system, with the springs in compression.

As before, wave-based control happens at a higher level, telling the actuator sub-controller how to move. A relatively simple arrangement gives very good results, as will be seen. It is organised as three 1-D WBC systems acting in parallel, one each for $x, y$ and $\theta$ motions, as in Fig. 1b. Each of these takes a reference or target displacement and first multiplies it by $1 / 2$. This half reference input becomes the "launch" wave that is the launch part of the actuator's motion for that component of the manoeuvre ( $x$-, $y$ - or $\theta$-motions). To these half-reference inputs are 
added the returning wave motions, $[x, y, \theta]_{\mathrm{b}}$. Just as with 1-D systems, all measurements are taken at the output of the actuator, where it interfaces with the flexible system. The returning translation "waves" in one direction are determined from both the actual actuator displacement in that direction and from the component of the interface force in that direction. The returning rotation "waves" are determined from the actuator rotation and from the moment of the interface forces about a fixed moment axis.

Measuring these returning waves in the 2D lumped model using wave transfer functions (WTFs), would face limitations and difficulties, as presented by O'Connor and Fumagalli [2]. For this reason, the simplest as well as the most efficient approach to measure has been adopted here in which these three returning waves are based on the force-impedance formulation. See [25]. So

$$
\begin{aligned}
& x_{b}=\frac{1}{2}\left[x-\frac{1}{Z_{x}} \int_{0}^{t} F_{x} d t\right], \\
& y_{b}=\frac{1}{2}\left[y-\frac{1}{Z_{y}} \int_{0}^{t} F_{y} d t\right], \\
& \theta_{b}=\frac{1}{2}\left[\theta-\frac{1}{Z_{\theta}} \int_{0}^{t} M d t\right]
\end{aligned}
$$

Here the $Z$ terms are impedances with constant values in a given control system. The actual value is not critical (see [4] for the robustness of WBC to $Z$ value): only the order of magnitude is significant. Varying the value provides some fine tuning of the final control system. $Z_{x}$ and $Z_{y}$ can be set to $\sqrt{ }(\mathrm{km})$, at least initially, where $k$ is a representative stiffness of the springs in contact with the actuator, and $m$ is a representative mass. In this work $Z_{\theta}$ was set to $\sqrt{ }(10 \mathrm{~km})$.

Then, for example, the $\theta$-motion component which the actuator sub-controller is asked to follow becomes

$$
\theta=\frac{1}{2} \theta_{\text {ref }}+\frac{1}{2}\left[\theta-\frac{1}{Z_{\theta}} \int_{0}^{t} M d t\right]
$$

As in the 1-D case, the addition of the returning wave components to the launch wave components of the actuator motion has two important effects. The first is that it causes the actuator to act like an active viscous damper for motion in the flexible system arriving back to the actuator. The equivalent viscous damping coefficients have values equal to the impedances. The second important effect is that, for a rest-to-rest manoeuvre, in the absence of external forces, when, say, $\theta_{\text {ref }}$ reaches its new value and becomes constant, the addition of the returning component quickly supplies the second half of the reference motion, causing the system to settle at the target position. In Eqs.(21) to (23) the force or moment integrals become zero on settling, because the initial and final momenta (linear or angular) are zero. Thus at the new steady state, $x_{s s}=1 / 2\left(x_{r e f}+x_{s s}\right)$ and so $x_{s s}=x_{r e f}$, as required. The same applies for the other two motion variables. In this way, planar position control and active vibration damping are seamlessly combined into a single motion of the actuator for all three aspects of the motion.

Regarding the rotational motion, if the point Q in Fig. 1b is taken as the moment axis, the control system using the rotational returning wave, Eq.(23), behaves exactly as required provided there is no simultaneous translation of the actuator. If however there is simultaneous translation, there will often be a small steady-state error in the final angle when the system settles. Obviously this could be corrected by a further rotational manoeuvre, but a more elegant solution is available. 
The source of the error is that the angular momentum of a system about some axis is not conserved, even in a rest-to-rest manoeuvre, if that axis moves in space (unless the axis is the system mass centre). So angular momentum about $Q$ is not necessarily conserved for a manoeuvre if Q moves, and so the integral in Eq. (24) does not return to zero exactly. The simplest way to avoid this (frequently small) error is to take moments, not about a moving axis such as $\mathrm{Q}$, but about a fixed axis, such as ' $\mathrm{O}$ ' in Fig. 1(b) which is the original position of $\mathrm{Q}$. Alternatively, one could take moments about the mass centre of the grid, requiring continual recalculation of its position and updating over time. Either solution ensures zero steady-state error in the final position. In either case, the system can then deal with simultaneous translational and rotational motion to a new target position with zero final error in all three target values.

As in the 1-D case, the shape of the reference inputs over time is arbitrary. They can be, for example, steps, ramps or s-shaped parabolic signals provided only that the final steady-state values correspond to the desired final position and orientation of the actuator. There is scope here to optimise the input waveforms, for example to achieve minimum time manoeuvres [3]. But simple inputs, such as ramps up to the target values, work so well in most cases that trying to do better is hardly worth the effort.

If, instead of the actuator, it is desired to get another part of the system, such as the tip, to a target position and orientation, it is a matter of simple geometry to adjust the target final actuator positions so that the part of the system of interest ends up in the position required, with the correct target angle. (Obviously if the only requirement is that the tip be at target $x$ and $y$ values, there is an infinite number of angular positions $\theta$ which would achieve the same position.)

\section{Cross coupling of motions}

In complex flexible systems such as the above-mentioned model, there is generally strong cross-coupling between translational and rotational motions. One of the motivations for this work, therefore, was to investigate how this cross coupling would affect the WBC in Fig. 1a, with its three parallel controllers, one for each aspect of the motion.

Consider, for example, the flexible array starting with $\theta=0$, with the free sides parallel to the $\mathrm{x}$ and $\mathrm{y}$ axes. Suppose it is desired to move it in the $y$-direction only (with no displacement in the $\mathrm{x}$ and $\theta$ directions). The actuator's $y$-motion has a shearing action on the flexible array, but it also has rotational (torque-like) effects, attempting, as it were, to keep the grid from rotating at the start and end of the manoeuvre. The actuator will experience a time-varying torque and it, in turn, is applying a torque in reaction.

To achieve this $y$-motion under $W B C$ there are at least two possibilities. In Fig. 1a one could use exclusively the $y$ component of the control system, with the $x$ and $\theta$ controllers simply turned off. In other words, the entire launching and absorbing motions of the actuator are exclusively in the $y$-direction. There might be a requirement to use such a control strategy if the actuator had only one degree of freedom, in the direction of motion, and so $x$ and $\theta$ motion were not available. As will be seen below, this simple, one-dimensional approach works as desired.

If instead one assumes that the actuator has all three degrees of freedom, a second control option is to launch the as before, but, in addition to absorbing again the measured returning $y$ motion, to measure and absorb simultaneously motions. When this is implemented, it gives better vibration control, but in general the final positions will be incorrect. Furthermore the final errors in the three variables are difficult to predict. The $x$ and $\theta$ absorbing take from the $y$ absorbing. It is as if the actuator finds the easiest and fastest way to absorb the vibration energy and momentum, using whatever variables it can control, but in doing so allows the three component motions to become mixed in an unpredictable way. For this reason this option is unlikely to be 
used in practice, unless rapid, active vibration damping takes absolute priority over position control.

A third option, which easily solves the mixing aspect, is to implement full control of all three variables, as in Fig.1a, even though the reference inputs to both the $\theta$ and $x$ motions would be zero in this case. The $\theta$ and $x$ controllers would still contribute to the absorbing, but now the reference input values (which happen to be zero) will ensure that the final values are correct. This third arrangement, as envisaged in Fig.1a, will generally be the default when $x, y$ and $\theta$ actuation are separately available. It gives very good results, as will be seen, combining good vibration control and zero settling error.

Similar cross coupling effects between component motions will arise in any given manoeuvre, involving changes in one, two or three of the motion variables. In all cases, the configuration of Fig. 1a gives the desired performance, simply and robustly. To illustrate the above some sample results will now be presented.

\section{Results}

A beam model, with no internal or external damping, was set up as shown in Figs. 1a. In the control system the measurements of force and displacement were obtained from the model springs and actuator position. The responses to various reference inputs were then investigated, with different levels of control in the three parallel aspects of the WBC system shown in Fig.1a.

The developed beam model, as Fig.1a depicting, consists of a $2 \times 5$ uniform grid geometry which makes it a relatively thin and long structure. The 20-DoF system has a bar-like actuator with a high degree of flexibility, especially for lateral and rotational movements. As an almost arbitrarily-chosen sample set of values, the system was given masses of $m=0.3 \mathrm{~kg}$ and three different spring constants, horizontal $\mathrm{k}_{\mathrm{x}}=200 \mathrm{~N} / \mathrm{m}$, vertical $\mathrm{k}_{\mathrm{y}}=400 \mathrm{~N} / \mathrm{m}$ and diagonal $\mathrm{k}_{\mathrm{d}}=300$ $\mathrm{N} / \mathrm{m}$, giving the beam model non-isotropic elastic properties. Also the sample beam was chosen to be $1.25 \mathrm{~m}$ long. The reference motion inputs are also arbitrary and are chosen to correspond to different trajectories in the plane that might be required in industry or under laboratory conditions. They can range from a simple translation to following a specific path combining 2$\mathrm{D}$ translational and rotational motions. Since the beam-shaped model has considerable longitudinal extension, it would be prone to slewing due to any $y$-direction translation, so such motions are more challenging from a control point of view compared to a longitudinal motion. Figure 4 depicts the response of the lumped beam model due to a 1-meter sliding in the $y$ direction through a ramp input waveform with rate of $1 \mathrm{~m} / \mathrm{s}$.

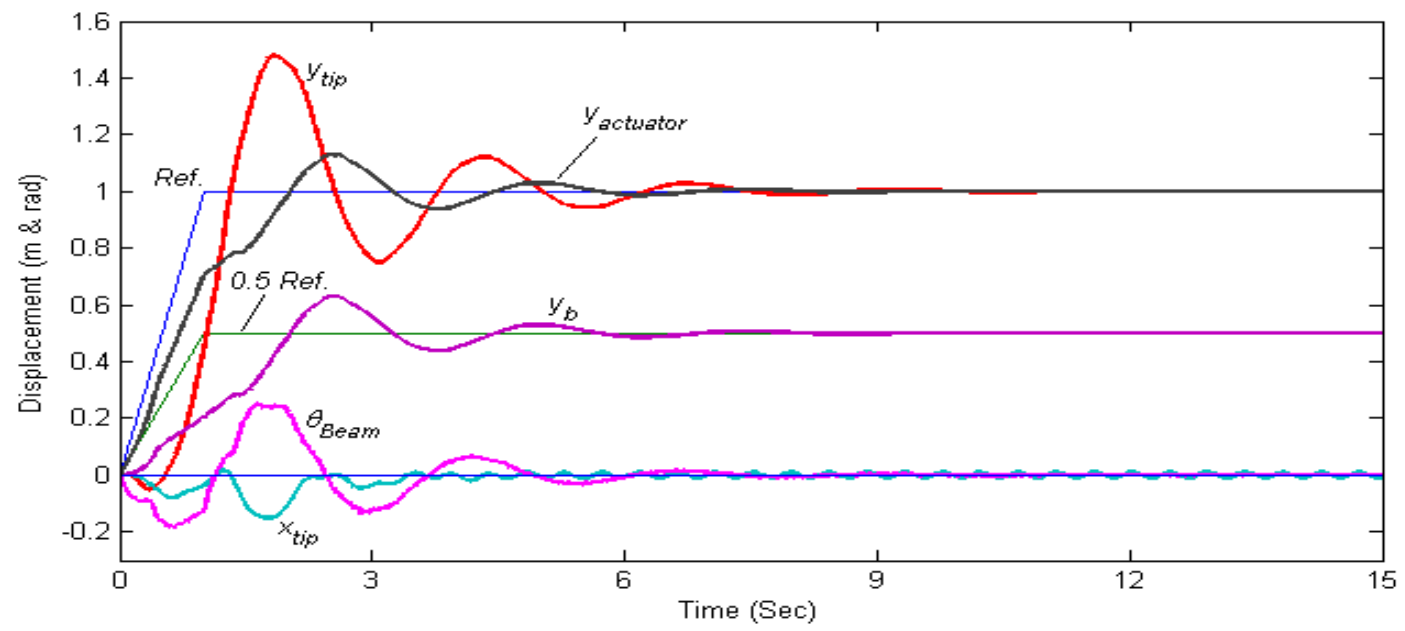

Fig. 4: System response for 1-m displacement in the direction y using only $y$-direction returning wave in the controller. 
Although the control of the system in Fig. 4 uses only y-returning motion absorption, the controller settles relatively quickly in this rest-to-rest manoevure. However, to improve the overall performance, including a reduction of the overshoot in the $y$ direction and suppression of the small lingering oscillations in the $x$ and $\theta$ directions, all three return motions should be absorbed. The results of this control implementation for the same manoeuvre as in Fig. 4 are shown in Fig. 5. The improvement is evident.

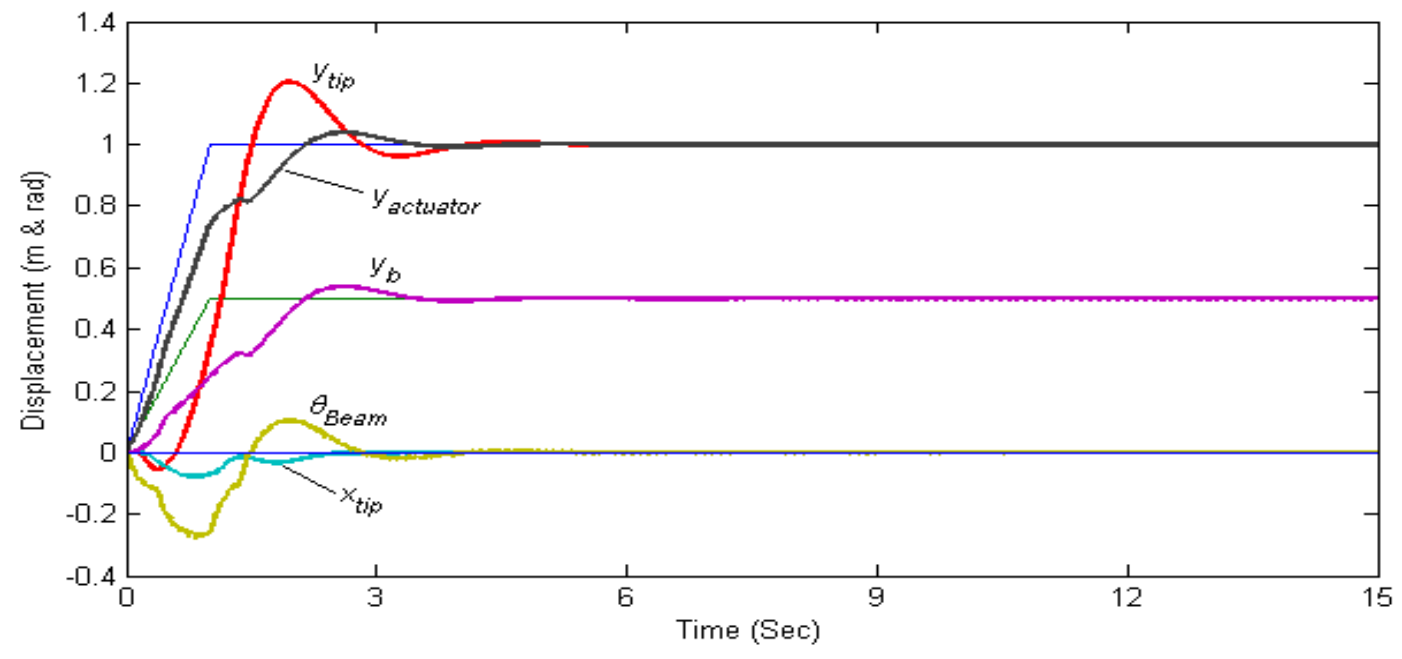

Fig. 5: The same maneuver as in Fig.4 but using $x, y$ and $\theta$ return waves in the controller.

In Figs. 4 and 5, the reference is a ramp to the target displacement of one unit in the $y$ direction. The launch wave component can be seen as exactly half this reference, while the measured return wave is labeled $y_{b}$. The sum of returning wave and the half reference becomes equal to the actual motion of actuator. The returning wave is a more dispersed and distorted version of the launch wave. The two-stage motion of the actuator is less clear, but WBC continues to work as designed. The blurring of the waveforms does not cause deterioration in the control performance.

In the following test, the tip is moved to be repositioned at a predefined target through a hybrid motion comprising a $1-\mathrm{m}$ translation in the $x$-direction along with a rotation of $\theta=0.4 \mathrm{rad}$. So in this manoeuvre, both $x$ and $\theta$ returning motions are necessarily added to the launching waves to ensure the desired displacements are achieved without error. However, the $y$-return motion has also been absorbed to improve the system response. As seen in Fig. 6, again the controller achieves a short transient time, small overshoot and rapid settling despite the model and manoeuvre complexities.

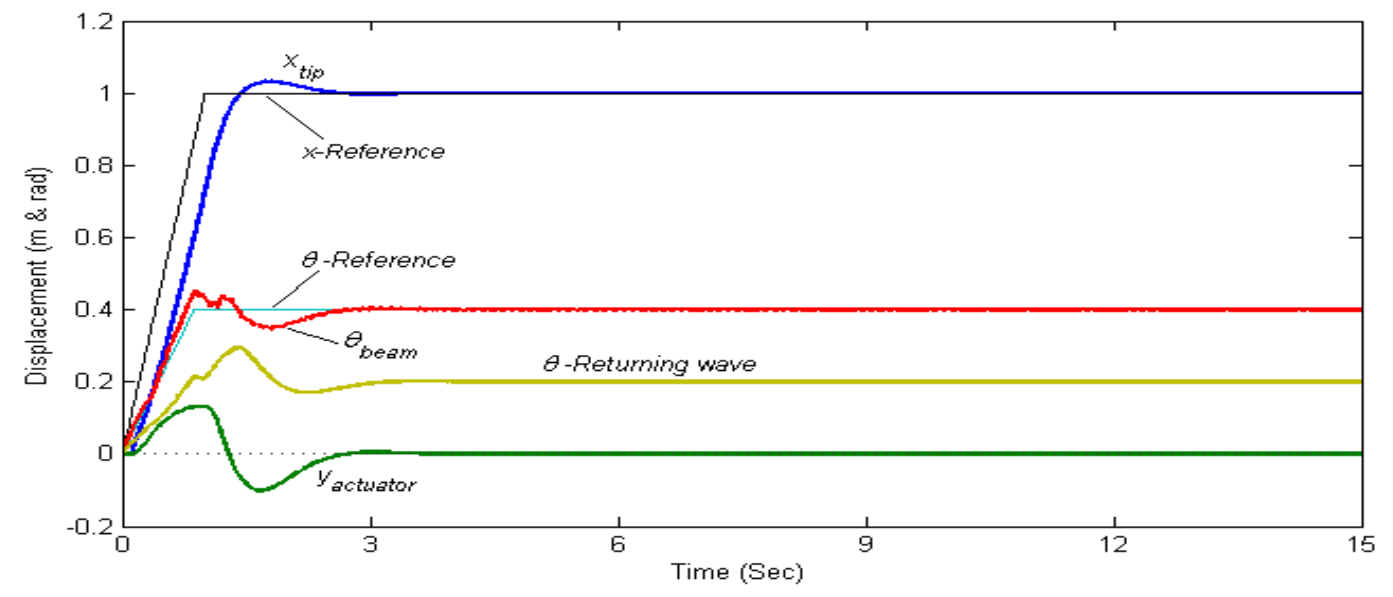

Fig. 6: WBC of a planar beam-like system to reach target through a combined motion (x-translation and rotation) using all returning waves. 
Compared to the last manoeuvre (shown in Fig. 5), less oscillation is seen here which is attributable to the absence of lateral motion ( $y$-direction).

As the most demanding test form the point of view of dynamical coupling, simultaneous rotation and planar translations in both the $x$ and $y$ directions were implemented. Target values were $x=1 \mathrm{~m}, y=0.8 \mathrm{~m}$ and $\theta=0.2 \mathrm{rad}$, created by three ramped inputs of different arbitrarilychosen slopes. Considering the longitudinally-extended beam and its high flexibility, this is perhaps the most challenging test, combining diverse reference motions at one time. Nonetheless, WBC effectively copes as before (see Fig. 7).

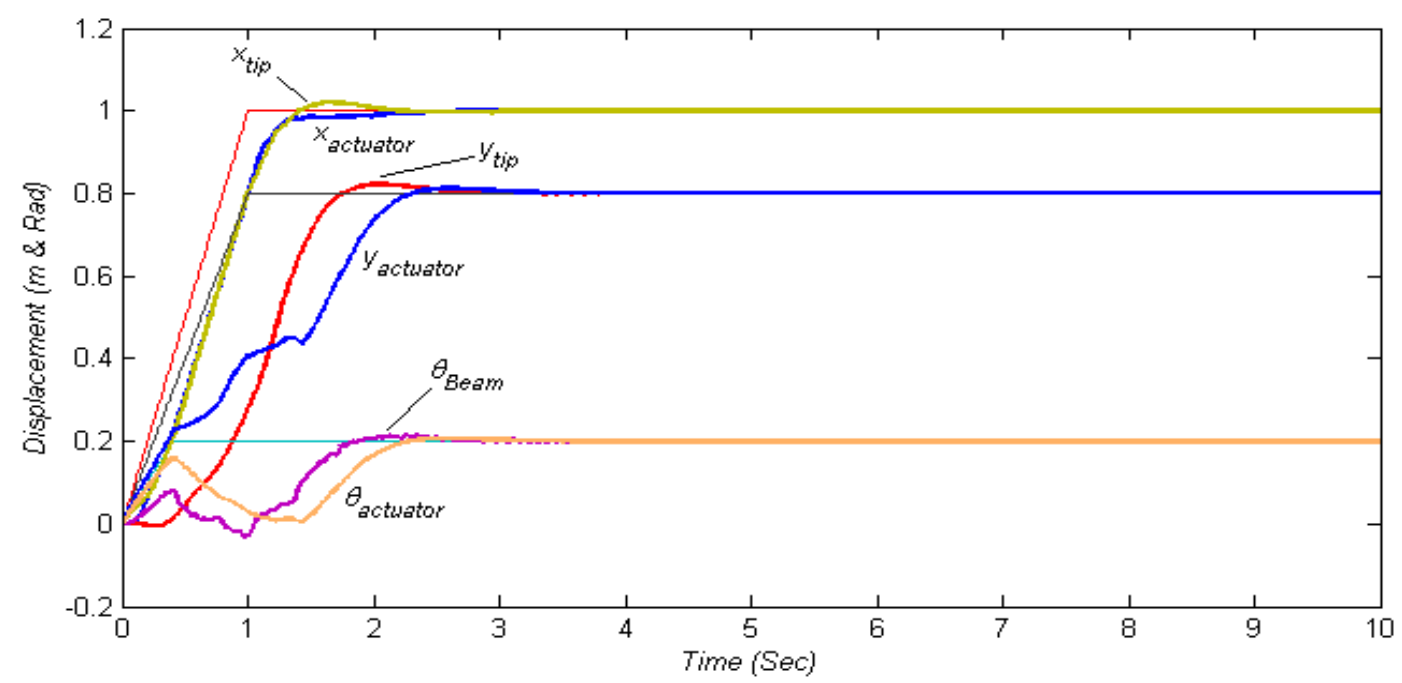

Fig. 7: WBC of the beam model for a complex input as three-component reference of $x, y$ and $\theta$

The next test, shown in Fig. 8, illustrates the robustness of the control system to changes in the reference inputs. Figure 8 depicts the response of the beam's tip to three ramp inputs, all up to a 1-m displacement in the $y$-direction, but with different velocities (gradients), using a same value of $\mathrm{Z}$. As can be seen, the controller of the 2-D beam model has a satisfactory performance in all three cases. The choice of ramp rate provides a way to achieve a trade-off between rise time, overshoot, and settling. In a given application one or more of these performance measures may take priority and the ramp rate can be chosen to suit the requirements. In any case, even with a steep ramp, the overshoot is relatively small, the settling time short, and there is zero steady-state error.

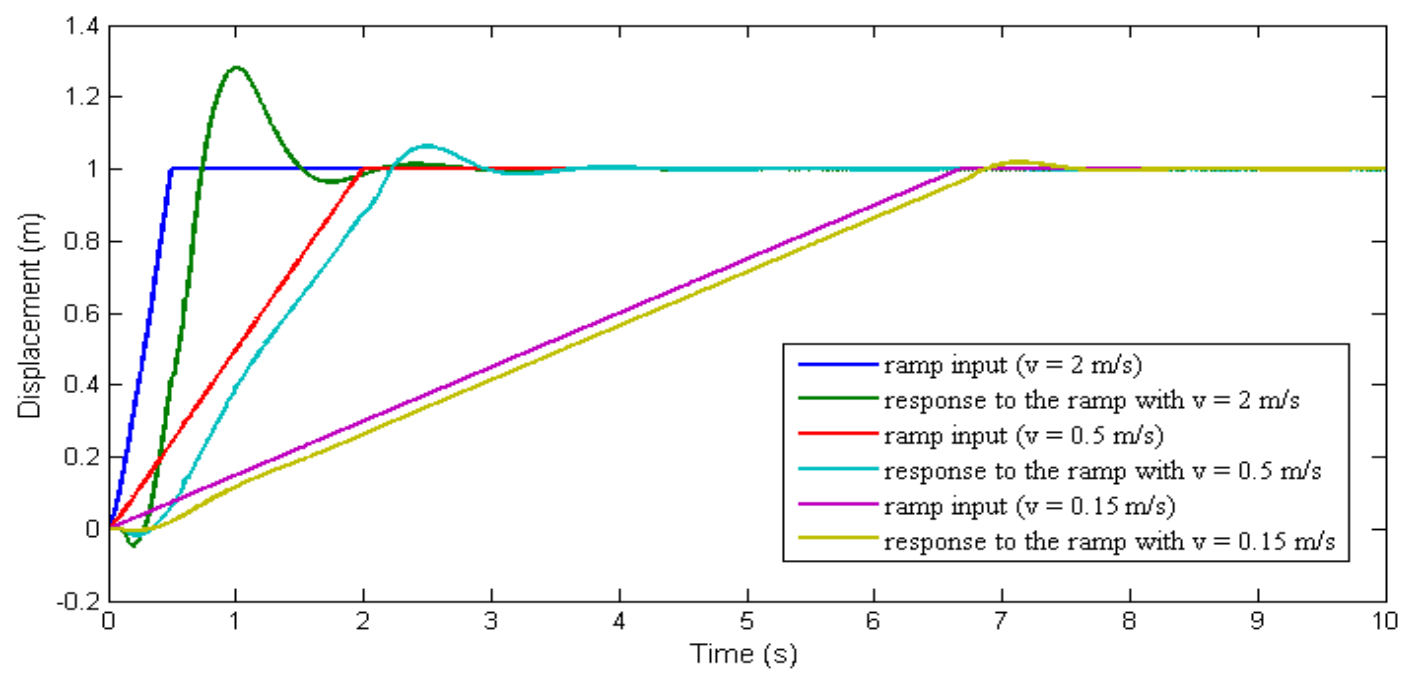

Fig. 8: Response of the beam tip to three ramp inputs to the same target $y$-displacement with different ramp rates (actuator velocities). 
Finally, to quantify how well WBC performs and to give some measure of the system flexibility and inherent periodic times, the responses of the system with no control is also shown in the last test presented in Fig. 9. For the results shown in Fig. 9, the target tip displacements (reference inputs) in the $x$ and $y$ directions were $0.4 \mathrm{~m}$ and $1 \mathrm{~m}$ respectively. The actuator was allowed motion only in the $x$ and $y$ directions, with all rotational $(\theta)$ motion suppressed. In other words, all absorbing of returning motion had to be achieved by $x$ and $y$ motions exclusively. The $x$ and $y$ reference inputs were simultaneous ramps of different slopes. As can be seen, also shown are the responses of the system (the $x$ and $y$ motions of the tip) for the same $x$ and $y$ reference inputs when no control is applied. The under-actuated, complex system is moved from rest to rest within about one period of its lowest mode of vibration.

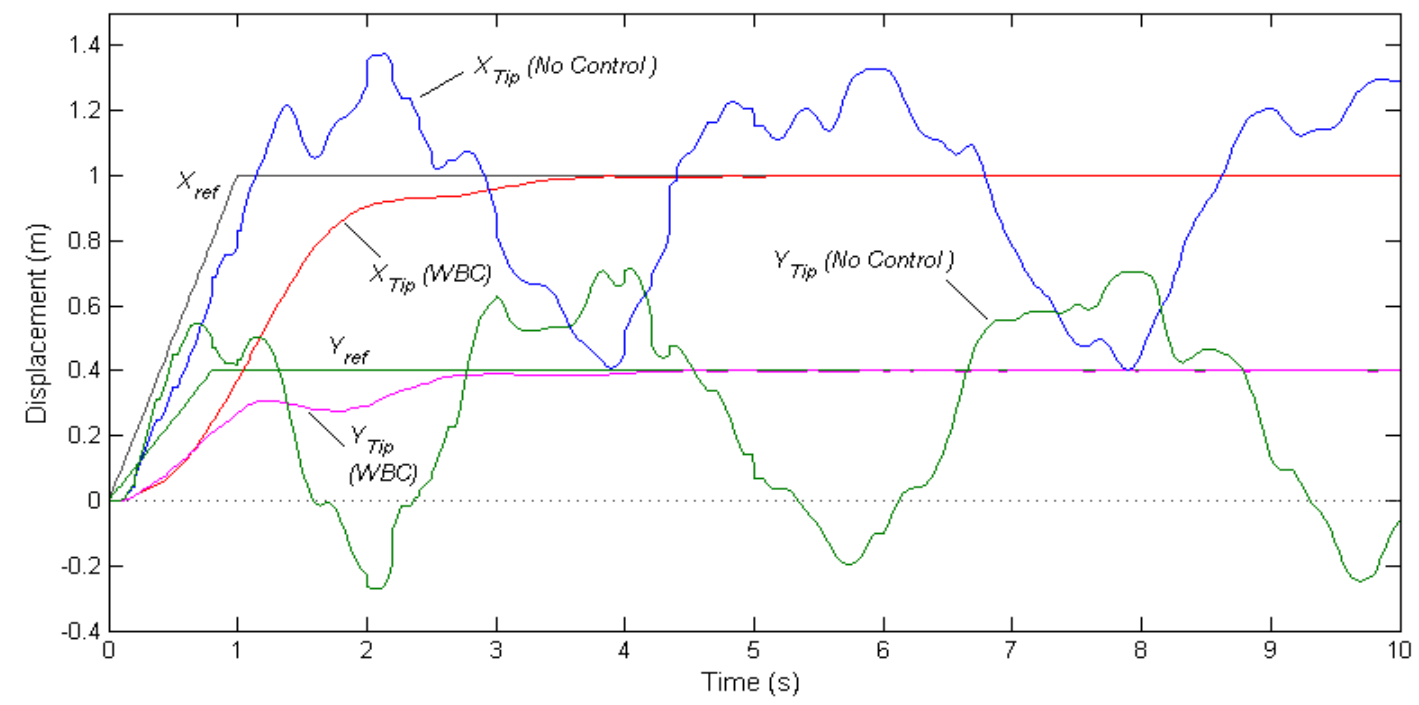

Fig. 9: Response of the beam model which moves in both $\mathrm{x}$ and $\mathrm{y}$ directions in a plane depicted for two separate situations: 1) applying WBC using same returning waves in the controller 2) No control applied

\section{Further remarks on results}

Although not shown here, other aspects were also investigated. The shape and arrangement of the mass-spring array was changed, for example to make it longer and thinner. Similarly satisfactory results were then obtained without having to change the control strategy. The satisfactory performance of the developed controller in these cases was interpreted by the system's responses of trivial overshoot and short settling time with no steady-state error similar to the results achieved and presented in the current paper.

Also the values of mass and spring elements were varied and made non-uniform, and again the same controller still gave very good results. This robustness to system changes is mainly due to the fact that the control law is based on the returning wave motion, which in turn is determined by the system dynamics, whatever they happen to be. The controller does not need to model them, or to know them. It simply waits, observes, and then moves to absorb them. This could be considered a form of real-time system identification.

The results above used an ideal actuator. To investigate the effects of non-ideal actuator response, the actuator was given second-order dynamics, giving rise to a delay between the actuator input and its response. Provided the bandwidth of the actuator extended to the highest frequency of the system, the WBC implementation still gave very good responses, similar to those shown. This latter aspect of the control system's robustness can be explained in part by the fact that the wave measurements are all based on the actuator's real motion (the motion it actually 
achieves) rather than on some theoretically desired motion. Also imperfect absorption of returning waves simply means that some small fraction of the returning wave, instead of being absorbed, will be reflected back into the system, but quickly finds its way back to the actuator where most of this residual motion is absorbed. A continuation of this process quickly absorbs most of the vibration, even with a non-ideal actuator.Regarding settling times, if standard definitions are used (e.g. time for the system to reach and stay within $5 \%$ or $2 \%$ of the final value) the settling times are very short.

\section{Concluding remarks}

The main conclusion of the present work is that, despite the added complexity of the dynamics of the 2-D system and its many degrees of freedom, a fairly simple generalisation of the WBC position control strategy proves to be very effective, retaining its many attractive features, including robustness to actuator limits, robustness to system changes, no requirement for a detailed system model, speed of response, small overshoot, rapid settling, zero steady-state error, all achieved with a simple, low-order controller. Although not presented here, the same system can also be used to model and control other types of flexible beams and plates, uniform or not, perhaps of irregular shapes, with non-isotropic elastic properties, as well as complex, elastic structures, lumped or continuous.

The chosen model of the flexible beam has many interesting features. It allows shear effects and centrifugal effects to be modelled naturally. It does not artificially separate so-called "rigid body" motion from flexible motion, and therefore does not raise concerns about unmodelled coupling effects between them. It is simple to implement, and can easily cope with non-uniform material properties and shapes. For present purposes it provides a useful test-bed for control ideas. But it has also been shown that, for beam-like structures, the lumped model gives comparable dynamic results to those from classical approaches to modelling distributed systems.

Finally, initial results soon to be reported suggest that the same techniques easily extends to the control of 3-D flexible systems, where the actuator has 6 degrees of freedom (3 translational and 3 rotational).

\section{References}

[1] W.J. O'Connor and D. Lang, Position Control of Flexible Robot Arms using Mechanical Waves. ASME Journal of Dynamics Systems, Measurement and Control, (1998) 120, no.3, pp.334-339.

[2] W.J. O'Connor and A. Fumagalli, Refined Wave-Based Control Applied to Nonlinear Bending and Slewing Flexible Systems. ASME Journal of Applied Mechanics, (2009) 76(4). doi:10.1115/1.3086434.

[3] W.J. O'Connor and D.J. McKeown, Time-optimal control of flexible robots made robust through wave-based feedback. ASME J Dynamic Systems, Measurement \& Control, (2011) Vol. 133, Issue 1.

[4] W.J. O'Connor and H. Habibi, Gantry crane control of a double pendulum, distributed-mass load, using mechanical wave concepts. Mechanical Sciences (2013) 4: 251-261. 
[5] H. Habibi and W.J. O'Connor, Payload motion control of rotary gantry and luffing cranes using mechanical wave concepts, Transactions of the Institute of Measurement and Control, SAGE journals, 2016, (online published); doi:10.1177/0142331216644044.

[6] H.G.Sage, M.F. de Mathelin and E. Ostertag, Robust control of robot manipulators: a survey. International Journal of Control, (1999) 72, 16, pp 1498-1522.

[7] R.D. Robinett III, C.R. Dohrmann, G.R. Eisler, J.T. Feddema, G.G. Parker, D.G. Wilson, and D. Stokes, Flexible robot dynamics and controls. (2002) Kluwer Academic/Plenum, New York.

[8] F.Y. Wang and Y. Gao, Advanced Studies of Flexible Robotic Manipulators: Modeling, Design, Control and Applications. World Scientific Publishing Company, (2003) ISBN-13: 9789812383907.

[9] M. Benosman and G.L. Vey, Control of flexible manipulators: asurvey. Robotica, (2004) 22, 5, pp 533-545.

[10] S.K. Dwivedya and P. Eberhardb, Dynamic analysis of flexible manipulators, a literature review. Mechanism and Machine Theory, (2006) 41, 7, pp 749-777.

[11] H.N. Rahimi and M. Nazemizadeh, Dynamic analysis and intelligent control techniques for flexible manipulators: a review. Journal of Advanced Robotics, (2014) Volume 28, Issue 2, 6376.

[12] W.J. O'Connor, Wave-Based Analysis and Control of Lump-Modelled Flexible Robots. IEEE Transactions on Robotics,(2007) 23, pp. 342-352.

[13] W.J. O'Connor, Wave-like modelling of cascaded, lumped, flexible systems with an arbitrarily moving boundary. Journal of Sound and Vibration (2011) 330: 3070-3083.

[14] B.R. Mace and R.W. Jones, Feedback Control of Flexural Waves in Beams, Journal of Structural Control, (1996) Vol. 3 N. 1-2, 89-98.

[15] C.M.A. Vasques and J.D. Rodrigues, Combined feedback/feedforward active control of vibration of beams with ACLD treatments: Numerical simulation, Computers and Structures 86 (2008), 292-306.

[16] S.M. Khot, N.P. Yelve, R. Tomar, S. Desai, and S. Vittal, Active vibration control of cantilever beam by using PID based output feedback controller. Journal of Vibration and Control. March 2012 vol. 18 no. 3, 366-372.

[17] S.H. Mirafzal, A.M. Khorasani and A.H. Ghasemi, Optimizing time delay feedback for active vibration control of a cantilever beam using a genetic algorithm, Journal of Vibration and Control, SAGE journals, 2015, (online published); doi: 10.1177/1077546315569863.

[18] F.-M. Li, G. Yao and Y. Zhang, Active control of nonlinear forced vibration in a flexible beam using piezoelectric material. Mechanics of Advanced Materials and Structures, Vol. 23, 2016 - Issue 3, pp 311-317.

[19] A. Eddanguir, and R. Benamar, A Discrete Model for Transverse Vibration of a Cantilever Beam Carrying Multi Lumped Masses: Analogy with the Continuous Model, Chapter: Design and Modeling of Mechanical Systems, Part of the series Lecture Notes in Mechanical Engineering, 2013, pp 89-96. 
[20] J.M. Gere and S.P. Timoshenko, Mechanics of Materials, (1997) PWS Publishing Company.

[21] M. Gams, M. Saje, S. Srpcic and I. Planinc, Finite element dynamic analysis of geometrically exact planar beams, Computers and Structures(2007) Vol. 85, 1409-1419.

[22] M. Cohodar, W. Borutzky, and V. Damic, Comparison of different formulations of 2D beam elements based on Bond Graph technique. Simulation Modelling Practice and Theory, (2009) Vol. 17, 107-124.

[23] J.H. Williams, Jr., Fundamental of applied dynamics, John Wiley \& Sons, 1996, USA.

[24] W.J. O'Connor and D.J. McKeown, A new approach to modal analysis of uniform chain systems, Journal of Sound and Vibration, 2008, 311(3-5), 623-632.

[25] W.J. O'Connor, Wave-echo control of lumped flexible systems, Journal of Sound and Vibration, (2006),Vol. 298, 1001-1018. 